\title{
Comparing Payments Between Sociobehavioral and Biomedical Studies in a Large Research University in Southern California
}

Journal of Empirical Research on Human Research Ethics 2021, Vol. 16(I-2) II7-124 DO! I0.1177/I556264620987773

journals.sagepub.com/home/jre

\section{Brandon Brown', Logan Marg', Emily Michels², Zhiwei Zhang', Dario Kuzmanović ${ }^{3}$, Karine Dubé ${ }^{4}$, and Jerome T Galea ${ }^{5}$}

\begin{abstract}
Given the dearth of regulatory guidance and empirical research on practices of providing payments to research participants, our study aimed to examine whether there were significant differences in payment amounts between sociobehavioral and biomedical studies and to examine study factors that may explain payment differences. This study reviewed 100 sociobehavioral and $3 \mathrm{I}$ biomedical protocols. Results showed that both biomedical studies and sociobehavioral studies had a wide variation of payments and, on average, the biomedical studies paid significantly more. Additionally, more biomedical studies offered payment than sociobehavioral studies. The primary factors that explained differences in payment amounts between sociobehavioral and biomedical studies were the number of study visits, study time, participation type, risk level, and research method. These findings provide pilot data to help inform future ethical decision-making and guidance regarding payment practices.
\end{abstract}

\section{Keywords}

payment for research participation, research ethics, behavioral social science research, federal policies/guidelines/office of human research protections, other behavioral/biomedical science

\section{Introduction}

Providing payments to research participants is a common practice (Dickert \& Grady, 1999). Researchers use payments to reimburse participants for expenses incurred through participation (e.g., transportation and parking), compensate the time and effort needed to participate, and bolster participant recruitment and retention (Anderson, 2019; Gelinas et al., 2018). Multiple models for payment exist, including treating participation as paid labor (Free Market Model, Wage Payment Model); compensating based on the theory that participants are partners in the research (Fair Share Model); and, reimbursing for out-of-pocket costs with only the potential for small tokens of appreciation, as research participation is considered an altruistic activity (Reimbursement Model, Appreciation Model) (Resnik, 2008). The ethical rationale behind payments for research participants is to ensure that there is a balance that allows participants' time, effort, and bodies to be valued but not commodified. Additionally, there must be ethical thought put into ensuring payments do not cause participants to overlook risks in order to receive reimbursement (Gelinas et al., 2018).
These payments raise ethical questions centered on the concepts of undue inducement, coercion, and exploitation-questions that cannot be answered without consensus on what constitutes undue inducement, coercion, and exploitation. For the purposes of this paper, we will use general definitions for these terms as follows: undue inducement refers to excessively attractive offers that lead people to do something to which they would normally have real objections based on their values or their risk assessments; coercion refers to an explicit threat of harm that is intentionally presented by one person to another to achieve compliance; exploitation refers to a situation whereby one party

\footnotetext{
'University of California, Riverside, USA

${ }^{2}$ Emory University, Atlanta, GA, USA

${ }^{3}$ University of Toronto, Canada

${ }^{4}$ University of North Carolina, USA

${ }^{5}$ University of South Florida, USA

Corresponding Author:

Brandon Brown, Center for Healthy Communities, School of Medicine, University of California, 3333 14th Street, Riverside, CA 9250I, USA. Email: brandon.brown@medsch.ucr.edu
} 
takes unfair advantage of another. Generally, undue inducement and coercion are regarded as invalidating informed consent either through primarily compromising (prospective) participants' voluntariness or comprehension of what their participation entails (Casarett et al., 2002; Largent et al., 2013, 2017). Many theorists conclude that since there is almost always the option to cease participation in a study and little risk of explicit threats of harm, coercion is very uncommon in clinical research (Grady et al., 2005). Others argue that payment-based coercion can exist in research, as "someone's interests can be partially set back in virtue of being subject to another's foreign will" (Millum \& Garnett, 2019). Undue inducement, by contrast, does not involve explicit threat, instead focusing on payment's ability to sway one's decision and, in this way, there is concern that payments to research participants could cloud their judgment, invalidating their informed consent to participate (Devine et al., 2015; Largent et al., 2017; U.S. Department of Health and Human Services, 2009).

There is also concern that payments to research participants can lead to exploitation if participants are not fairly compensated for their time and effort. A broader expression of this concern regards the disproportionate burdens of research borne by people of low socioeconomic status, while people of higher socioeconomic status reap the benefits (Denny \& Grady, 2007; Elliot \& Abadie, 2008; Largent et al., 2019; Smith, 2018). In the extensive literature exploring these concerns, scholars continue to debate whether and when payments to research participants result in undue inducement, coercion, and/or exploitation, the meaning of such concepts, and generally what constitutes and how to determine appropriate payments (Grady, 2019; Largent et al., 2017, 2019; Malmqvist, 2019; Millum \& Garnett, 2019).

In the midst of these ongoing ethical debates, laws, regulations, and ethical guidelines do little to provide substantive or practical guidance to researchers and ethics committees about payments to research participants. Existing sets of guidelines tend to provide more general guidance on payment-for example, the importance of ensuring the amount is not too high or too low (The Belmont Report of the USA), or maintaining sensitivity to the economic circumstances of the potential participants (TCSP2 of Canada) - but not specific information on appropriate ranges of payment or factors that can influence the amount of compensation. Moreover, ethical debates and researchers' and ethics committees' decisions about payments occur against a backdrop of scant empirical evidence.

Additional empirical evidence is needed describing payment amounts in actual studies and examining study-related factors associated with payment amounts. As expectations of research participants differ based on numerous factors including culture, history, location, and the type of research being conducted, it is also important to gather information on payment variation between categories of studies (e.g., collection of self-reported data vs. physiological testing or collection of biological data) to examine the balance of risk and reward. Although empirical evidence has its limitations - especially due to the extreme variations in payment structures based on a multitude of factors - such evidence could inform ongoing ethical debates and the development of empirically derived institutional guidance regarding payments to research participants (e.g., helping establish payment benchmarks for different types of research and research tasks) (Anderson, 2019; Brown et al., 2018; Brown et al., 2019). The present paper addresses gaps in the literature by comparing payments between sociobehavioral and biomedical studies at a single study site and identifying study factors that may explain payment differences. The comparison of sociobehavioral and biomedical studies allows for the evaluation of different types of research that are typically associated with lower and higher risk assessments, respectively. As discussed later in the paper, participants are exposed to varying levels of risk based on the study methods and the categorization of study; focusing on the sociobehavioral and biomedical categories allows for a straightforward representation of lower and higher research risk profiles.

\section{Methods}

We conducted a cross-sectional, retrospective review of Institutional Review Board (IRB)-approved sociobehavioral and biomedical research protocols and related study materials (i.e., IRB protocols, amendments, informed consent forms, correspondence between IRB analysts, and primary investigators) between 2017 and 2020 at a large research university in Southern California. This study was exempt from IRB review because it neither interacted with study participants nor collected identifiable data. The university's institutional official gave permission for access to the administrative data.

We collected the following data: type of study (sociobehavioral or biomedical), study risk level (minimal or more than minimal), number of participant visits, time commitment, research method (qualitative, quantitative, or mixed), participation type (remote, in-person, or both), population type (college students, non-student adults, children, or multiple), whether payment was offered (something or nothing), monetary payment amount (in U.S. dollars), and payment form (money, academic credit, or other). At this institution's IRB office, sociobehavioral studies are defined as those that "involve research on individual or group characteristics and may entail collection of data by interviews, focus groups, use of video or audio recordings, or research with identifiable dataset." For clinical or biomedical studies, any component of skin penetration, application of heat, cold, or electric/electromagnetic fields would establish the study as biomedical and therefore reviewed by the 
Table I. Factors Associated with Payments to Research Participants.

\begin{tabular}{|c|c|c|c|c|}
\hline \multirow[b]{2}{*}{ Variable } & \multicolumn{2}{|c|}{ Socio-behavioral studies } & \multicolumn{2}{|c|}{ Biomedical studies } \\
\hline & $\begin{array}{c}\text { Number of studies } \\
\text { (\% providing payment) } N(\%)\end{array}$ & $\begin{array}{c}\text { Payment amount } \\
\text { mean|median (range) }\end{array}$ & $\begin{array}{c}\text { Number of studies (\% } \\
\text { providing payment) N (\%) }\end{array}$ & $\begin{array}{c}\text { Payment amount } \\
\text { mean|median (range) }\end{array}$ \\
\hline & $100(55)$ & $15.92 \mid 0.00(0-225)$ & $31(74)$ & $136.16 \mid 0.00$ (0-2880) \\
\hline \multicolumn{5}{|l|}{ Visits } \\
\hline 1 & $66(54.5)$ & $8.20 \mid 0.00(0-100)$ & $15(73)$ & $24.33 \mid 20.00(0-75)$ \\
\hline 2 & $12(66.7)$ & $25.02 \mid 1.62(0-120)$ & $7(57)$ & $23.00 \mid 6.00$ (0-115) \\
\hline$>2$ & $20(55)$ & $37.50 \mid 0.00(0-225)$ & $9(89)$ & $4|0.56| 120.00(0-2880)$ \\
\hline \multicolumn{5}{|l|}{ Time commitment } \\
\hline 30 minutes or less & $22(72.7)$ & $4.67 \mid 0.75(0-30)$ & $4(50)$ & $25.00 \mid 15.00(0-70)$ \\
\hline$>30-60$ minutes & $35(45.7)$ & $2.97 \mid 0.00(0-50)$ & $5(40)$ & $4.00 \mid 0.00(0-20)$ \\
\hline$>60$ minutes & $40(57.5)$ & $34.62 \mid 0.00(0-225)$ & $20(95)$ & $205.05 \mid 40.00$ (0-2880) \\
\hline \multicolumn{5}{|l|}{ IRB review type } \\
\hline Exempt & $5(40.0)$ & $3.40 \mid 0.00(0-15)$ & $0(0)$ & NA \\
\hline Expedited & $93(55.9)$ & $16.66 \mid 0.00(0-225)$ & $29(72)$ & |32.96|20.00 (0-2880) \\
\hline Full review & $2(50.0)$ & I2.50|| $2.50(0-25)$ & $2(100)$ & $|82.50| 182.50$ (II5-250) \\
\hline \multicolumn{5}{|l|}{ Population type } \\
\hline Non-college adults & $58(43.1)$ & $15.29 \mid 0.00(0-225)$ & II (45) & $286.36 \mid 0.00$ (0-2880) \\
\hline College students & $22(68.2)$ & $10.68 \mid 0.00(0-60)$ & $10(90)$ & $37.50 \mid 20.00$ (0-225) \\
\hline Multiple & $13(92.3)$ & $25.00 \mid 5.00(0-120)$ & $9(89)$ & $74.00 \mid 40.00(0-250)$ \\
\hline Children & $6(50.0)$ & $24.17 \mid 0.00(0-120)$ & I (100) & $30.00 \mid 30.00(30-30)$ \\
\hline Organization & I $(0.0)$ & $0.00 \mid 0.00(0-0)$ & $0(0)$ & NA \\
\hline \multicolumn{5}{|c|}{ In-person and/or remote participation } \\
\hline In-person & $55(47.3)$ & $19.54 \mid 0.00(0-225)$ & $27(78)$ & I55.00|20.00 (0-2880) \\
\hline Remote & $27(70.4)$ & $3.95 \mid 0.25(0-50)$ & $2(50)$ & $15.00 \mid 15.00(0-30)$ \\
\hline Both & $18(55.6)$ & $22.79 \mid 2.50(0-120)$ & $2(50)$ & $3.00 \mid 3.00(0-6)$ \\
\hline \multicolumn{5}{|c|}{ Research method category } \\
\hline Quantitative & $46(69.6)$ & $18.12 \mid 0.62(0-225)$ & $29(72)$ & |4I.59|20.00 (0-2880) \\
\hline Qualitative & $39(28.2)$ & $7.31 \mid 0.00(0-120)$ & $0(0)$ & NA \\
\hline Mixed & $15(80.0)$ & $33.43 \mid 6.00(0-200)$ & $2(100)$ & $57.50 \mid 57.50(40-75)$ \\
\hline
\end{tabular}

biomedical IRB. For biomedical studies, we collected additional data on the type of study procedure that participants experienced during the research (i.e., brain scan, blood draw, other specimen collection). The number of study visits was defined as any researcher-participant contact in order to encompass research with physical study visits or those that did not involve face-to-face contact (e.g., online surveys). Additionally, as some study protocols included a range of possible payments, study visits, and time commitments, each of these variables was operationalized as the maximum possible for the single study under question. In other words, if a study paid a different amount based on number of visits, then the maximum payment amount was reported for that study.

The association between study type (sociobehavioral or biomedical) and other study characteristics was tested individually (one at a time) using Chi-squared test (for categorical variables) or logistic regression. Logistic regression was also used to model the probability of paying study participants as a function of study type and other study characteristics. We further conducted log-linear regression models with generalized estimating equations to determine whether there were significant differences in payment amounts between sociobehavioral and biomedical studies and to examine study factors that may explain payment differences. Analyses were conducted using R version 3.5.2.

\section{Results}

We reviewed 100 sociobehavioral and 31 biomedical protocols (Table 1). Monetary payments in biomedical studies ranged from $\$ 0$ to $\$ 2,880$ (average $\$ 136$ ), while in sociobehavioral studies payment ranged from $\$ 0$ to $\$ 225$ (average $\$ 15.90)$. Although a larger proportion of biomedical studies offered payment than sociobehavioral studies $(74 \%$ vs. $55 \%)$, the difference was not significant $(p=.09)$. On average, biomedical studies paid significantly more than sociobehavioral studies $(p=.002)$, with sociobehavioral studies paying an average of $11.7 \%$ of biomedical studies. Compared to sociobehavioral studies, biomedical studies typically required more time (median 90 vs. 60 minutes) and more visits (median 2 vs. 1), and tended to be more 
Table 2. Logistic Regression for Dichotomous Payment Status.

\begin{tabular}{llcc}
\hline Variable & \multicolumn{1}{c}{ Level } & Odds ratio & $95 \% \mathrm{Cl}$ \\
\hline Study type & Clinical (referent) & & $(0.33,4.09)$ \\
Visits & Non-clinical & 1.17 & $(0.95,1.06)$ \\
Log2(time) & & 1.00 & $(0.99,1.93)$ \\
Participation type & Both (referent) & 1.39 & .9640 \\
& In-person & 2.40 & .0544 \\
Remote & Mixed (referent) & $(0.66,8.69)$ & $(0.84,22.25)$ \\
& Qualitative & & $(0.01,0.37)$ \\
Research method & Quantitative & 0.07 & $(0.13,2.86)$ \\
& Minimal (referent) & 0.62 & .0003 \\
& More than minimal & 7.54 & $(0.67,84.86)$ \\
\hline
\end{tabular}

Table 3. Log-Linear Regression for the Continuous Payment Amount.

\begin{tabular}{|c|c|c|c|c|}
\hline Variable & Level & Mean ratio & $95 \% \mathrm{Cl}$ & $p$-value \\
\hline \multirow[t]{2}{*}{ Study type } & Clinical (referent) & & & .6931 \\
\hline & Non-clinical & 0.90 & $(0.52,1.55)$ & \\
\hline Visits & & 1.02 & $(1.02,1.03)$ & $<.0001$ \\
\hline Log2(time) & & 1.28 & $(1.15,1.43)$ & $<.0001$ \\
\hline \multirow[t]{3}{*}{ Participation type } & Both (referent) & & & .0087 \\
\hline & In-person & 2.31 & $(0.97,5.53)$ & \\
\hline & Remote & 0.68 & $(0.19,2.36)$ & \\
\hline \multirow[t]{3}{*}{ Research method } & Mixed (referent) & & & .0270 \\
\hline & Qualitative & 0.21 & $(0.07,0.66)$ & \\
\hline & Quantitative & 0.66 & $(0.35,1.23)$ & \\
\hline \multirow[t]{2}{*}{ Risk level } & Minimal (referent) & & & .0120 \\
\hline & More than minimal & 2.22 & $(1.19,4.15)$ & \\
\hline
\end{tabular}

quantitative (94\% vs. 46\%), more in-person (87\% vs. 55\%), and of greater risk (19\% vs. $2 \%$ with more than minimal risk). The two types of studies did not differ substantially in population type and IRB review type. In a logistic regression model relating payment to study type and other study characteristics, the only significant predictor was research method $(p<.001)$, with estimated odds ratios of 0.62 (95\% CI: $0.13-2.86)$ for quantitative versus mixed and of 0.07 (95\%CI: $0.01-0.37)$ for qualitative versus mixed (Table 2).

In a log-linear regression model relating payment amount to study type and other study characteristics, study type was non-significant $(p=.69)$. Significant predictors included number of visits $(p<.001)$, study time $(p<.001)$, participation type $(p=.009)$, risk level $(p=.012)$, and research method $(p=.027)$ (Table 3$)$.

\section{Discussion}

This study compared payments between sociobehavioral and biomedical studies and examined study factors that explained differences. We found that both biomedical studies and sociobehavioral studies had a wide variation of payments and, on average, the biomedical studies paid significantly more. Additionally, more biomedical studies offered payment than sociobehavioral studies. The primary factors that explained differences in payment amounts between sociobehavioral and biomedical studies were number of study visits, study time, participation type, risk level, and research method. An alternative explanation is that biomedical studies are more well-funded compared to sociobehavioral studies.

Consistent with previous research, the wide variation of payments within biomedical and sociobehavioral studies suggests that payment decisions are relatively unstructured in both types of studies, which is expected given the dearth of institutional and regulatory guidance on payments to research participants (Bernstein \& Feldman, 2015; Grady et al., 2005; Henderson, 2015; Largent et al., 2017). Still, our findings suggest that researchers' payment decisions are influenced by the type of research (e.g., quantitative, qualitative, or mixed), the amount of time that participation 
requires, the amount of risk that participation entails, and the number of interactions between participants and researchers, rather than whether the research was biomedical or sociobehavioral, which is consistent with previous research (Fry et al., 2005; Grady et al., 2005; Marathe et al., 2018; Ripley et al., 2010a). The present study is also consistent with previous research that suggests that biomedical studies typically pay more than sociobehavioral studies and that such differences may be due to greater risk in biomedical studies, which our study also showed (Grady et al., 2005).

The Office for Human Research Protections (OHRP) notes that "remuneration to subjects may include compensation for risks associated with their participation in research" (Office for Human Research Protections, 2019); however, regulatory guidelines are often vague on how risk level, and other study factors we evaluated, should affect payments (Gelinas et al., 2018). When evaluating research studies for IRB approval, payment is not to be considered a benefit in the weighing of risks and benefits, so it cannot serve the purpose of justifying the study's IRB-identified risks. The IRB does take into consideration the payment amount and rationale in order to make sure that these factors will not reduce participant decision-making capacity or informed consent (U.S. Food \& Drug Administration, 2018). Some IRBs/RECs support specific payment models, such as the endorsement of payment for time, inconvenience, and expenses (e.g., South Africa's National Health Research Ethics Council) (NHREC, 2012). These specific practical guidelines are scarce, however. Interestingly, in a survey of IRB chairpersons and investigators, there are clear inclinations toward specific payment models used to determine payment amounts - the Reimbursement Model (41.6\%) and Market Model (32.7\%) taking the primary spots, respectively - although these are not necessarily reflected in official regulations (Ripley et al., 2010b).

The finding that higher risk studies paid more than lower risk studies raises concerns about the potential for undue influence (Resnik, 2015). Such concerns may be particularly salient because the university from which we collected our data lacked uniform standards or guidance for determining appropriate payments. Some scholars, noting the lack of consensus about when payments could result in undue influence, argue that payments should not be based on the level of risk that study participation entails (Cleary et al., 2016; Ensign, 2003; Hammett \& Sporton, 2012). Others argue that it is acceptable for researchers to base payment amounts at least partially on risk level, because concerns about undue influence should be largely mitigated so long as there is a well-functioning, independent ethical review board (e.g., IRB), which is charged with doing a proper risk-benefit assessment and minimizing risks and burdens (Emanuel, 2005; Largent et al., 2013, 2017, 2019). As a part of undue influence, some ethicists are concerned that payment allows potential participants to obscure or ignore potential risks. One study of risk versus payment could not substantiate the claim that increased payment clouded risk assessment (Bentley \& Thacker, 2004); another study found that higher payment studies were perceived as higher risk, leading to participants spending more time learning about the riskier studies and weighing the consequences of involvement (Cryder et al., 2010). The concern remains, however, especially for low-income participants who may be more inclined to accept risk or endure clinical procedures that they ordinarily would not seek out in exchange for higher payment (Denny \& Grady, 2007).

While we cannot determine the rationales behind payment decisions with a retrospective review of administrative data, our findings showing that payments depended on time required for participation, number of interactions between researchers and participants, and that the majority of biomedical and sociobehavioral studies offered payments suggest researchers were aware of the importance of compensating research participants for their time and effort, which may reduce worries of exploitation (Largent et al., 2013, 2017, 2019). Still, the question remains about whether payments were adequate/appropriate enough: in some cases, payments may have been too low and therefore potentially exploitative. Exploitation, in the context of research payments, can be present even if both parties - researcher and participant — are gaining benefits and have given consent for the terms of the transaction. For example, although a participant receives payment, the institution carrying out the research may stand to benefit, financially or academically, exponentially more than the participant (Resnik, 2015). While it is implausible and categorized as undue influence to compensate participants at a level that would match the benefit to the researchers, there must be attention paid to determining fair and balanced compensation.

\section{Best Practices}

The wide variation of payments within biomedical and sociobehavioral studies suggests that payment decisions are relatively unstructured in both types of studies. As a general recommendation, we support the collection of more data on the topic, both qualitative and quantitative, to inform the development of ethical guidelines and IRB decisions. As such, we recommend that study authors not only give more specific information on what payments or non-monetary types of compensation were given, but also describe their reasoning behind the payments they provided in their peer reviewed manuscripts. Doing so would provide a starting point for gathering more data on how payment decisions are made in both biomedical and sociobehavioral studies. Currently, rationales behind payment are seldom, if ever, reported or requested. Understanding how payment 
decisions are made is important for generating practical guidance, especially given the differences between payment in biomedical and sociobehavioral research.

This study's findings should be considered alongside its limitations. While we reviewed 100 sociobehavioral studies (the number of studies approved in a single year) and all the available biomedical study protocols over several years at one institution $(N=31)$, our sample size was relatively small. As such, our findings may not generalize to other institutions or non-university contexts with significantly more biomedical research. Some of the differences in the literature could also be due to how studies define sociobehavioral and biomedical research, with some defining biomedical research as clinical trials (phases I-IV) only. Additionally, we could not identify rationales behind payment decisions, because justification was requested but not provided. Finally, many of the reviewed study protocols included a range of possible payments, study visits, and time commitments, and we used the maximum possible value in our analysis. Payments may have also excluded non-monetary types of compensation, such as complimentary transportation to study sites or parking vouchers, which when paired with monetary payment could translate to higher payment amounts.

\section{Research Agenda}

Future research should carefully consider payment and the impact that study risk has on payment decisions, both for the researcher and the participant. Participant expectations of payment should also be explored further, including the effects of previous research experience, identification with a specific patient community, specific local conditions, and burden versus risk of a study (Devine et al., 2015; Grady et al., 2005). It may also be worthwhile to complete a review of the effect that available funding has on the distribution of payments to research participants, as well as the effect it has on the recruitment of participants (Largent \& Lynch, 2017). While some scholars are currently working to establish some guidance for payment, IRBs should do more to try to collect data on reasoning for payments and decision making. Given the gap in payment decisions in biomedical and sociobehavioral research, and differences in funding amounts, more attention to the payment topic is needed.

\section{Educational Implications}

Guidance for payment in research should be provided as part of IRB applications. This guidance may not be study specific, but it may provide ranges of current and previous research with various study factors including risk, study type, and study setting which in of itself is a form of guidance. Doing so would enable key stakeholders in the research process - including funders, policy makers, study participants, ethics committee members, and researchersto critically assess how payment decisions are made, how they report payment data, and how data can be used to develop payment policy. Since local institutions may be competing for participants in similar research projects, standards of payment may be important for equity in research. While we have reported all clinical research here in a single institution, more institutions should be comparing biomedical and sociobehavioral payment data to better understand if higher study risk leads to higher payment.

\section{Conclusion}

We demonstrated differences in payments between biomedical and sociobehavioral research and identified study factors that explained such differences. Our study highlights the possible role of study risk or invasiveness in study payment decisions. This relationship is significant even though regulations state that payment should not be used as a method to compensate for risk, as payment is not considered to be a benefit to participants by IRB. Our findings should be used to inform continued scholarly debate about payments to research participants (e.g., what constitutes appropriate payments), as well as provide direction to empirically informed institutional guidance for research participant payments.

\section{Acknowledgments}

We'd like to thank the IRB analysts, Monica Wicker and Lorraine Castro, who assisted with and provided access to the data. We thank Jo Gerrard and Elizabeth Mendez-Nova for editorial assistance.

\section{Declaration of Conflicting Interests}

The author(s) declared no potential conflicts of interest with respect to the research, authorship, and/or publication of this article.

\section{Funding}

The author(s) disclosed receipt of the following financial support for the research, authorship, and/or publication of this article: This study was supported in part by a National Institutes of Health diversity supplement (3R01AI114617-03S1).

\section{References}

Anderson, E. E. (2019). A proposal for fair compensation for research participants. The American Journal of Bioethics, 19(9), 62-64.

Bentley, J. P., \& Thacker, P. G. (2004). The influence of risk and monetary payment on the research participation decision making process. Journal of Medical Ethics, 30(3), 293-298. https://jme.bmj.com/content/30/3/293. https://doi. org/10.1136/jme.2002.001594 
Bernstein, S. L., \& Feldman, J. (2015). Incentives to participate in clinical trials: Practical and ethical consideration. The American Journal of Emergency Medicine, 33(9), 1197-1200.

Brown, B., Galea, J. T., Dube, K., Davidson, P., Khoshnood, K., Holtzman, L., Marg, L., \& Taylor, J. (2018). The need to track payment incentives to participate in HIV research. IRB: Ethics \& Human Research, 40(4), 8-12. https://www.ncbi. nlm.nih.gov/pubmed/30387975

Brown, B., Marg, L., Zhang, Z., Kuzmanovic, D., Dube, K., \& Galea, J. (2019). Factors associated with payments to research participants: A review of sociobehavioral studies at a large Southern California Research University. Journal of Empirical Research on Human Research Ethics, 14(4), 408-415.

Casarett, D., Karlawish, J., \& Asch, D. A. (2002). Paying hypertension research subjects. Journal of General Internal Medicine, 17(8), 650-652.

Cleary, M., Siegfried, N., Escott, P., \& Walter, G. (2016). Super research or super-researched?: When enough is enough. Issues in Mental Health Nursing, 37(5), 380-382.

Cryder, C. E., London, A. J., Volpp, K. G., \& Loewenstein, G. (2010). Informative inducement: Study payment as a signal of risk. Social Sciences and Medicine, 70(3), 455-464.

Denny, C. C., \& Grady, C. (2007). Clinical research with economically disadvantaged populations. Journal of Medical Ethics, 33(7), 382-385. https://www.ncbi.nlm.nih.gov/ pubmed/17601862. https://doi.org/10.1136/jme.2006.017681

Devine, E. G., Knapp, C. M., \& Sarid-Segal, O, et al. (2015). Payment expectations for research participation among subjects who tell the truth, subjects who conceal information, and subject who fabricate information. Contemporary Clinical Trials, 41, 55-61.

Dickert, N., \& Grady, C. (1999). What's the price of a research subject? Approaches to payment for research participation. New England Journal of Medicine, 341(3), 198-203. https://www.ncbi.nlm.nih.gov/pubmed/10403861. https://doi. org/10.1056/NEJM1

Elliot, C., \& Abadie, R. (2008). Exploiting a research underclass in phase 1 clinical trials. New England Journal of Medicine, 358(22), 2316-2317. https://www.ncbi.nlm.nih.gov/pubmed/ 18509119. https://doi.org/10.1056/NEJMp0801872

Emanuel, E. J. (2005). Undue inducement: nonsense on stilts? The American Journal of Bioethics, 5(5), 9-13. Discussion. W18$11, \mathrm{~W} 17$.

Ensign, J. (2003). Ethical issues in qualitative health research with homeless youths. Journal of Advanced Nursing, 43(1), 43-50.

Fry, C. L., Ritter, A., Baldwin, S., Bowen, K. J., Gardiner, P., Holt, T., Jenkinson, R., \& Johnston, J. (2005). Paying research participants: A study of current practices in Australia. Journal of Medical Ethics, 31(9), 542-547. https://www.ncbi. nlm.nih.gov/pubmed/16131558. https://doi.org/10.1136/ jme.2004.009290

Gelinas, L., Largent, E. A., Cohen, G., Kornetsky, S., Bierer, B. E., \& Lynch, H. F. (2018). A framework for ethical payment to research participants. The New England Journal of Medicine, 378(8), 766-771.

Grady, C. (2019). The continued complexities of paying research participants. The American Journal of Bioethics, 19(9), 5-7.
Grady, C., Dickert, N., Jawetz, T., Gensler, G., \& Emanuel, E. (2005). An analysis of U.S. practices of paying research participants. Contemporary Clinical Trials, 26(3), 365-375. https://www.ncbi.nlm.nih.gov/pubmed/15911470. https://doi. org/10.1016/j.cct.2005.02.0

Hammett, D., \& Sporton, D. (2012). Paying for interviews? Negotiating ethics, power and expectation. Area, 44(4), 496-502.

Henderson, G. E. (2015). The ethics of HIV "cure: Research: What can we learn from consent forms? AIDS Research and Human Retroviruses, 31(1), 56-63.

Largent, E. A., Emanuel, E. J., \& Lynch, H. F. (2019). Filthy lucre or fitting offer? Understanding worries about payments to research participants. The American Journal of Bioethics, 19(9), 1-4.

Largent, E. A., \& Fernandez Lynch, H. (2017). Paying research participants: Regulatory uncertainty, conceptual confusion, and a path forward. Yale Journal of Health Policy, Law, and Ethics, 17(1), 61-141.

Largent, E. A., Grady, C., Miller, F. G., \& Wertheimer, A. (2013). Misconceptions about coercion and undue influence: Reflections on the views of IRB members. Bioethics, 27(9), 500-507.

Largent, E. A., \& Lynch, H. F. (2017). Paying research participants: The outsized influence of "undue influence." IRB, 39(4), 1-9.

Malmqvist, E. (2019). "Paid to endure": Paid research participation, passivity, and the goods of work. The American Journal of Bioethics, 19(9), 11-20.

Marathe, P. A., Tripathi, R. K., Shetty, Y.C., Kuyare, S.S., Kamat, S. K., \& Thattle, U. M. (2018). Payment for participation in clinical research: Review of proposals submitted to the ethics committees. Perspectives in Clinical Research, 9(2), 64. https://doi.org/10.4103/picr.PICR_159_16

Millum, J., \& Garnett, M. (2019). How payment for research participation can be coercive. The American Journal of Bioethics, 19(9), 21-31.

National Health Research Ethics Council (NHREC). (2012). Payment of trial participants in South Africa: Ethical considerations for research ethics committees. http://nhrec.health. gov.za/index.php/grids-preview?download=11:guidelinesfor-payment

Office for Human Research Protections. (2019). Attachment A - addressing ethical concerns offers of payment to research participants. https://www.hhs.gov/ohrp/sachrp-committee/recommendations/attachment-a-september-30-2019/index.html

Resnik, D. B. (2008). Increasing the amount of payment to research subjects. Journal of Medical Ethics, 34(9), e14.

Resnik, D. B. (2015). Bioethical issues in providing financial incentives to research participants. Medicolegal and Bioethics, 5, 35-41.

Ripley, E., Macrina, F., Markowitz, M., \& Gennings, C. (2010a). Who's doing the math? Are we really compensating research participants? Journal of Empirical Research on Human Research Ethics, 5(3), 57-65. https://www.ncbi.nlm.nih.gov/ pubmed/20831421. https://doi.org/10.10.1525/jer.2010.5.3.57

Ripley, E., Macrina, F., Markowitz, M., \& Gennings, C. (2010b). Why do we pay? A national survey of investigators and IRB chairpersons. Journal of Empirical Research 
on Human Research Ethics, 5(3), 43-56. https://www. ncbi.nlm.nih.gov/pmc/articles/PMC3168552/. https://doi. org/10.1525/jer.2010.5.3.43

Smith, J. (2018). Parasitic and parachute research in global health. Lancet Global Health, 6(8), E838. https://doi.org/10.1016/ S2214-109X(18)30315-2.

U. S. Department of Health and Human Services. (2009). Code of federal regulations - Title 45 Public Welfare CFR 46. https:// www.hhs.gov/ohrp/sites/default/files/ohrp/policy/ohrpregulations.pdf

U.S. Food \& Drug Administration. (2018). Payment and reimbursement to research subjects: Guidance for Institutional Review Boards and Clinical Investigators. https://www.fda. gov/regulatory-information/search-fda-guidance-documents/ payment-and-reimbursement-research-subjects

\section{Author Biographies}

Brandon Brown is an associate professor of medicine at the University of California, Riverside. His interest in the ethics of payment in research arose from his experience conducting clinical trials. He supervised the stellar graduate who collected the data for this manuscript and provided guidance in manuscript preparation.

Logan Marg is a recent sociology $\mathrm{PhD}$ graduate at the University of California, Riverside. He has worked on numerous research projects related to the ethics of payment in research with Dr. Brown. He collected the data, contributed to the analysis, and drafted the manuscript.
Emily Michels is a health policy analyst and bioethics researcher. She received her Masters of Bioethics from Emory University in May 2020 with a focus on the ethics of HIV genetic sequencingbased interventions, community engagement, and responsible policy development. She reviewed the paper and made final additions.

Zhiwei Zhang is an associate professor of biostatistics at the University of California, Riverside. He is interested in developing statistical methods for biomedical applications, including causal inference and precision medicine. He contributed to the analysis and interpretation of data in the present research.

Dario Kuzmanović is a research ethics enthusiast with 20 years of experience in research. He currently works at University of California, Riverside and is a member of the Joint Centre for Bioethics at University of Toronto. In the present research, he assisted with study design, data analysis, and manuscript preparation.

Karine Dubé is an assistant professor in the Public Health Leadership Program (PHLP) in the Gillings School of Global Public Health at the University of North Carolina at Chapel Hill . She works with Dr. Brown on the ethics of incentives in HIV research. She reviewed the paper for intellectual contents.

Jerome T Galea is an assistant professor in the School of Social Work at the University of South Florida. His interest is on the impact of incentives on research participation and how investigators arrive at payment amounts. His collaboration in this project included instrument development, data analysis, and manuscript preparation. 